\title{
Topic: INGUINAL HERNIA - Joined guidelines
}

\author{
(C) Springer-Verlag 2014
}

\section{PO:140}

\section{COMBINED INGUINAL HERNIA,} A PROGRESSIVE PROTRUSION DISEASE

G. Amato', G. Romano', A. Agrusa', G. Di Buono', G. Cocorullo', G. Gulotta ${ }^{1}$

'Department of General Surgery and Emergency University Palermo, Palermo, ITALY

Introduction: Combined hernia represents an almost frequent type of inguinal hernia protrusion. It is characterized by the merging of a direct and indirect hernia that develops through the inguinal floor. Nevertheless, regarding its classification there is a certain degree of confusion. Further uncertainty exists in determining how the protrusions confluence and progress. This investigation aims to clarify these matters thus enabling a better understanding of its development. The results of the research could result helpful in finalizing updated surgical techniques for a suitable management of this intricate anatomical condition.

Methods: The occurrence of combined hernias in the last 100 groin hernia repair procedures carried out by a single operator represent the source of the investigation. Photographic documentation reported in the course of inguinal hernia repair procedures highlights its gross anatomical characteristics. Looking forward to assess the pathway of this type of protrusion, these image data were crossed with the histological findings of biopsies excised during surgery.

Results: The results of the investigation were very interesting. The photographic documentation showed combined inguinal hernia types at different phases of expansion. Starting from the progenitor of the combined hernia, the pantaloon hernia, all steps of the combined hernia development were highlighted. Among these, noteworthy is the variant known as tricomponent protrusion, composed by a hernia of the supravescical fossa plus a direct and an indirect hernia. Histology highlighted a broad range of progressive damage involving the structure of this part of the abdominal wall. Macroscopic evidences of structural degeneration of the internal ring, progressive dissolution of the inguinal floor, disbanding until complete dissolution of the inferior epigastric vessels, adipose dystrophy of the muscle fibers and severe nerve damages were histologically documented.

Conclusion: The analysis of macro and microscopic data provided in the study supports the hypothesis that combined hernia represents the end stage of a progressive disease. Actually, these findings seem to confirm that inguinal hernia is a never-ending pathological condition produced by chronic compressive structural damage as consequence of a continuous visceral impact upon the inguinal floor. Determining the progressive character of this hernia type can result useful in better classifying all steps of hernia protrusion, thus allowing the development of improved surgical techniques and updated devices for inguinal hernia repair.

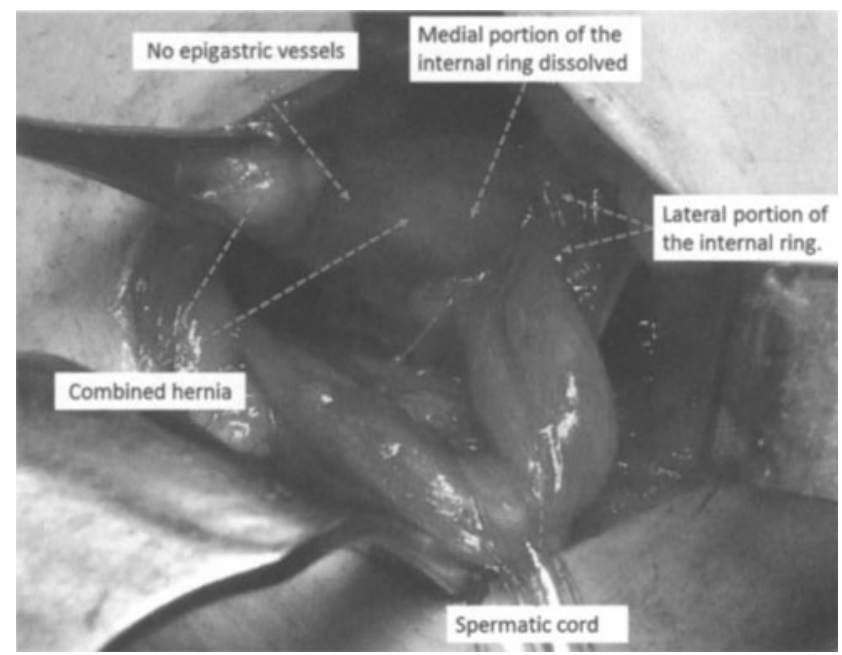

